\title{
THE COMPARATIVE EFFECTS OF CONTINUOUS AND INTERMITTENT PENICILLIN THERAPY ON THE FORMATION OF ANTISTREPTOL- YSIN IN HEMOLYTIC STREPTOCOCCAL PHARYNGITIS ${ }^{1}$
}

\author{
By EDWIN D. KILBOURNE 2, 3 AND J. PHILIP LOGE 4 \\ (From the Fort Monmouth Station Hospital, Fort Monmouth, N. J.; and the First Army \\ Laboratory, New York City) \\ (Received for publication January 8, 1948)
}

In the Spring of 1947 an epidemic of streptococcal pharyngitis and scarlet fever attacked a large permanent army post $^{5}$ numbering about eight thousand troops. A carrier survey just prior to this outbreak had disclosed a high rate (17 per cent) of carriers of beta hemolytic streptococci. Study of these organisms and those isolated from patients during the epidemic revealed that all were of Lancefield Group A. Seven-eighths of those isolated from carriers, and all streptococci isolated from patients, were either type 23 or 19 . Infections caused by type 23 predominated and constituted about 70 per cent of all cases.

During a 60-day period, 184 patients received a preliminary diagnosis of streptococcal pharyngitis or scarlet fever and were placed in a special study group at the time of their admission to the post station hospital.

The objectives of this study were: (1) to evaluate and delineate the natural history of streptococcal pharyngitis in the young male, (2) to assess the value of three treatment schedules (detailed below), and (3) to determine and evaluate the antistreptolysin antibody response of patients in each of the three treatment groups.

This paper will be concerned only with the third objective of this study.

\section{OUTLINE OF THE INVESTIGATION}

\section{Laboratory methods}

The following studies were made of each patient, usually within 24 hours of admission: (1) Total leukocyte

1 The authors wish to acknowledge their indebtedness to M/Sgt. R. J. Helmold who supervised the antistreptolysin titration and to Mrs. Florence Kessler, Bacteriologist, for their invaluable cooperation in this study.

2 Capt., M.C., AUS.

3 Now an Assistant of the Rockefeller Institute for Medical Research, 66th Street and York Avenue, New York 21, N. Y.

4 Dept. of Medicine, Washington University, St. Louis.

5 Fort Monmouth, New Jersey. count, (2) urinalysis, (3) chest X-ray, (4) Dick test, (5) antistreptolysin titer, and (6) throat culture.

Subsequently, the total leukocyte counts were repeated on the fourth and ninth days; urinalysis and Dick test were repeated on the ninth day; throat cultures were repeated on the fourth, ninth, and 21st days, and the antistreptolysin titer was determined on the 21 st day following admission.

Throat cultures were obtained as single specimens without the use of special methods or media. A cotton swab was touched to the tonsils and posterior pharynx, then immersed in brain-heart infusion broth, where it remained at room temperature until streaked over horse blood agar. After incubation, plates were studied with respect to the proportion of colonies producing beta hemolysis. No detailed colony counts were made, but all plates were evaluated by the same experienced bacteriologist with respect to predominance of beta hemolytic organisms. Cultures were reported as showing "predominating," "mixed," or "no significant" growth on blood agar. Organisms showing beta hemolysis on the first and final cultures were isolated in pure culture and forwarded to the First Army Laboratory for grouping and typing.

Antistreptolysin titration was performed by $\mathrm{M} /$ Sgt. R. J. Helmold using a modification of Todd's (1) original method. This modification entails the addition of 50 cc. of a Dextrose-Phosphate buffer to each liter of sterile Todd-Hewitt broth, and the use of $\mathrm{M} / 25$ Cystiene $\mathrm{HCl}$ as a streptolysin reducing agent (added to the streptolysin just prior to its use). A two-tube rise in titer has been regarded as significant. A fall in titer has not been accepted as evidence of a recent infection.

\section{Preliminary selection of patients}

During the period of study one or both of the authors personally examined in the hospital receiving office virtually all patients admitted with fever or complaints referable to the upper respiratory tract. Those suspected of streptococcal pharyngitis on the basis of previously defined clinical criteria were dispatched in approximate alternate rotation to one of several "study wards."

Although the work of the Commission on Acute Respiratory Disease (2) has emphasized the difficulty of establishing a clinical diagnosis of streptococcal pharyngitis, Rantz, et al. (3) have stated that such a .diagnosis may be made with reasonable accuracy. With the use of criteria similar to those of Rantz, an attempt was made to 
differentiate streptococcal sore throat from "non-specific pharyngitis" during a period when 516 cases of upper respiratory infection were admitted to the hospital. Of this number, 184 were separated from the others by the following criteria :

\section{Clinical criteria}

(1) History: The patients usually gave a history of the rapid onset of malaise, sore throat, chilliness or shaking chills. Vomiting, moderate prostration, and generalized aching were frequently present.

(2) Physical signs: The pharynx, tonsils and palate were invariably red and edematous, although the edema was often slight. Edema of the uvula proved almost pathognomonic. The soreness of the throat was often evident in the cautious manner in which the patient opened his mouth. The presence of exudate had been anticipated as a diagnostic key, but this finding was often absent. When present, it was green and confluent. Tender cervical adenitis, especially of the tonsillar nodes, was sought for and was usually evident.

The oral temperature was usually at least 100 degrees F. and prostration was sometimes severe.

A frank scarlatinal rash was of obvious diagnostic value and was considered indicative of streptococcal infection when present.

\section{SEPARATION AND TREATMENT OF PATIENTS}

At the commencement of the study, virtually all of 54 patients selected by these clinical criteria were treated with customary penicillin dosage, i.e., 20-50,000 units every three hours for a period of four to seven days (this schedule is hereafter referred to as Penicillin II). Subsequently, however, the remaining patients $(130)$ were assigned alternately to one of two study wards; in one, antimicrobic therapy was administered and in the other no specific treatment was given. With the exception of two patients suffering from peritonsillar abscess at the time of admission, separation of patients into "treated" and "untreated" groups was effected without regard for the severity of the disease. Ward officers on the "untreated". wards were not permitted to initiate penicillin therapy without consulting one of the investigators. This point is stressed because in the available literature on the therapy of streptococcal pharyngitis the establishment of a large, strict control group, untreated with sulfonamides or salicylates, has not been reported. Most authors acknowledge that therapy was usually given the most severely ill, and that such therapy was often at the discretion of ward surgeons.

\section{"Untreated" group}

The patients in this group received symptomatic therapy in the form of hot saline gargles or irrigations, obligatory bed rest for three days, and codeine when necessary. Salicylates were specifically interdicted in order to permit accurate observation of fever duration. All patients were closely watched for the development of complica- tions. Two who developed peritonsillar abscesses were started on penicillin therapy and were eliminated from this group. (The comparative results of penicillin and symptomatic treatment will be described elsewhere [4].)

\section{"Treated" groups}

The patients in these groups received the symptomatic therapy outlined above, including the obligatory period of bed rest. In addition, they received treatment with one of two penicillin regimens, which for convenience will be denoted Penicillin I and Penicillin II.

Penicillin I: This schedule entailed a single daily injection of 300,000 units of penicillin in aqueous solution for six to seven successive days. The rationale for this unusual and extravagant dosage will be presented later.

Penicillin II: Patients in this group (already mentioned) received penicillin in dosages comparable to usual experience; 20-50,000 units every three hours for from four to seven days. Most of the patients so treated (75 per cent) received penicillin for six or more days. The average period of therapy was 5.6 days, and the usual individual dose was 30,000 units. Selection of this regimen was predicated on the experience of Plummer and his co-workers, who found that clinical relapse occurred frequently in patients treated less than six days (5). According to Rammelkamp and Kirby (6), 20,000 units of penicillin "produces concentrations having maximal anti-streptococcal action for a period of more than two and one-half hours with partially inhibitory levels for another one and one-half to two hours." Thus, this dosage plan provided almost continuous levels effective against the hemolytic streptococcus.

\section{DEFINITIVE DIAGNOSIS}

It was realized from the outset that the diagnosis of streptococcal pharyngitis is difficult, and that there exist differences of opinion regarding the necessity of demonstrating a rise in the antistreptolysin titer for proof of diagnosis. The recent studies of Weinstein have indicated that in scarlet fever treated with long term (tenday) penicillin therapy there is usually no antistreptolysin response (7). Thus, in an undoubted streptococcal infection, there is evidence that penicillin therapy may suppress antibody response. As it was realized that in the present study those cases which were treated with penicillin might show fewer instances of antibody response, rigid and exacting criteria for the final inclusion of patients in this series were established regardless of how clinically "typical" they had been on admission. The criteria were: (1) history and physical findings compatible with a diagnosis of streptococcal pharyngitis (as delineated earlier in this paper), (2) fever of greater than 100 degrees $\mathrm{F}$. on the day of admission, (3) predominating growth of beta hemolytic streptococci on the initial throat culture, and (4) leukocytosis; i.e., a total leukocyte count greater than 10,000 cells per cu. mm.

The few cases which have been included which did not necessarily fulfill all four of these criteria, were cases in 
which: (a) a significant antistreptolysin rise was demonstrated, or $(b)$ the patient had a scarlatinal rash, or (c) peritonsillar abscess from which hemolytic streptococci were cultured existed at the time of admission.

The criteria were probably unduly severe. Plummer et al. noted that "an unexpected feature of (severe hemolytic streptococcal pharyngitis) was the slight increase or absence of increase in the total white blood cell count (5)." Rantz and his associates state that leukocytosis of "diagnostic value" is seen in only 49.1 per cent of patients (3) and that fever may be absent in patients with streptococcal pharyngitis (8). It seemed desirable to include only indisputable cases, however, in a study in which an important criterion of diagnosis was often lacking.

The application of the criteria plus the loss of patients by transfer and incomplete laboratory data reduced the series from 184 to 127 patients. The antistreptolysin rise in the "untreated" group (see Figure 1), however, affirms the accuracy of the preliminary diagnoses in a high proportion of instances.

\section{RESULTS}

The antistreptolysin response in patients receiving no specific treatment

In the group receiving no specific treatment, which included 51 patients, 84.3 per cent demonstrated a significant rise in antistreptolysin titer (Figure 1). This incidence is comparable to the value of other observers $(9,10)$. The average amplitude of the antistreptolysin rise was 462

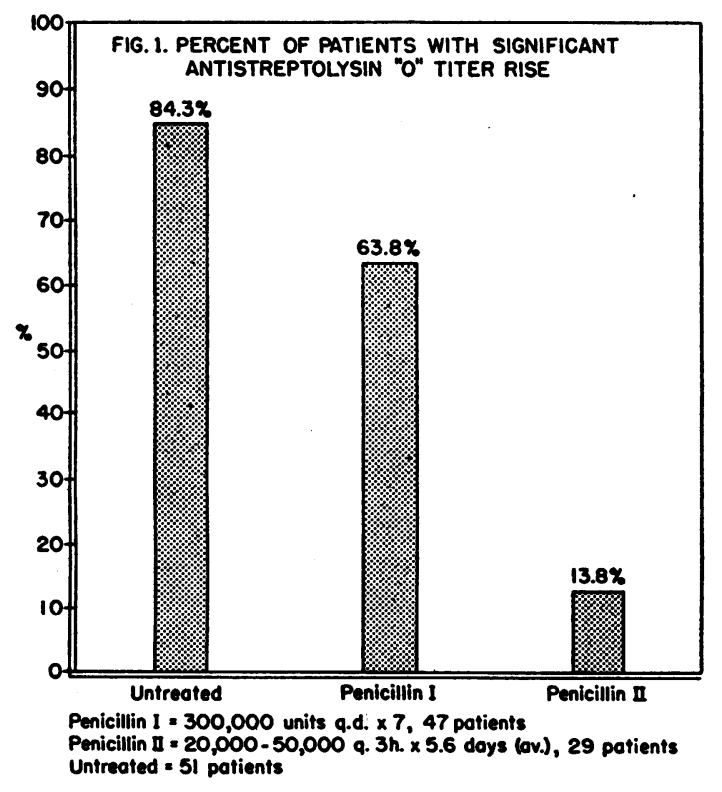

FIG. 1

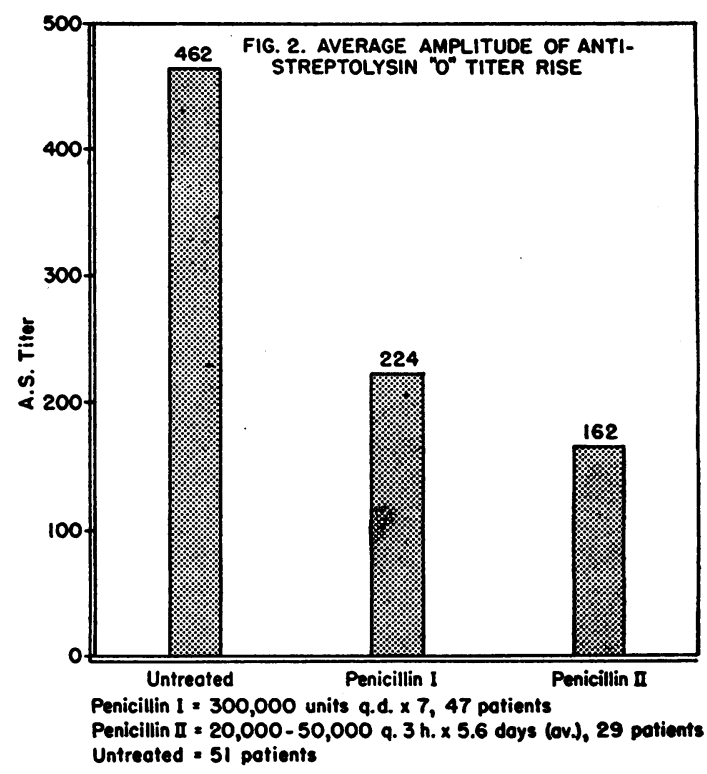

FIG. 2

units (Figure 2). The greatest rise (1150 units) is graphically shown in Figure 3.

The antistreptolysin response in patients receiving Penicillin Dosage I (large single daily dose)

63.8 per cent of 47 patients treated with Penicillin Dosage I exhibited a significant rise in antistreptolysin titer (Figure 1). This rise was of less amplitude than in the "untreated" group, averaging 224 units in patients with significant antistreptolysin responses (Figure 2). The extreme rise was 1050 units, comparable to that in the untreated group (Figure 3 ).

\section{The antistreptolysin response in patients receiving Penicillin Dosage II (small frequent doses)}

Only 13.8 per cent of the 29 patients treated with Penicillin Dosage II manifested a significant antistreptolysin rise (Figure 1). This rise was the smallest seen, and averaged 162 units in the four patients showing antibody response (Figure 2). The maximum rise exhibited by a patient in this group was only 300 units (Figure 3 ).

\section{DISCUSSION}

The results of the investigation indicate striking suppression of the incidence and degree of antibody formation by continuous penicillin treat- 
ment and remarkably little inhibition of antibody response by intermittent penicillin therapy.

The apparent suppression of antibody formation in the penicillin-treated groups must be critically evaluated. It is essential to establish that patients in these groups had actually suffered streptococcal infection. The typical clinical appearance of the patients, and the exacting criteria used in their definitive diagnosis have been described. As some investigators believe that a rise in antistreptolysin titer is requisite proof of infection by the hemolytic streptococcus, reference is made to the high percentage rise in the "untreated" control group. In this series of alternately selected cases a similar rise is predictable in the penicillin-treated groups.

The percentages of patients exhibiting low concentrations of antistreptolysin were analyzed because Rantz has stated that the height of the initial level of antistreptolysin may affect the incidence or degree of rise in titer (11).

In the "untreated" group 68.3 per cent had initial levels of 100 units or less of antistreptolysin. In the group showing the least number of antistreptolysin responses (Penicillin II) the incidence of low initial levels was 65.5 per cent. Thus this factor may scarcely be construed as an important source of error.

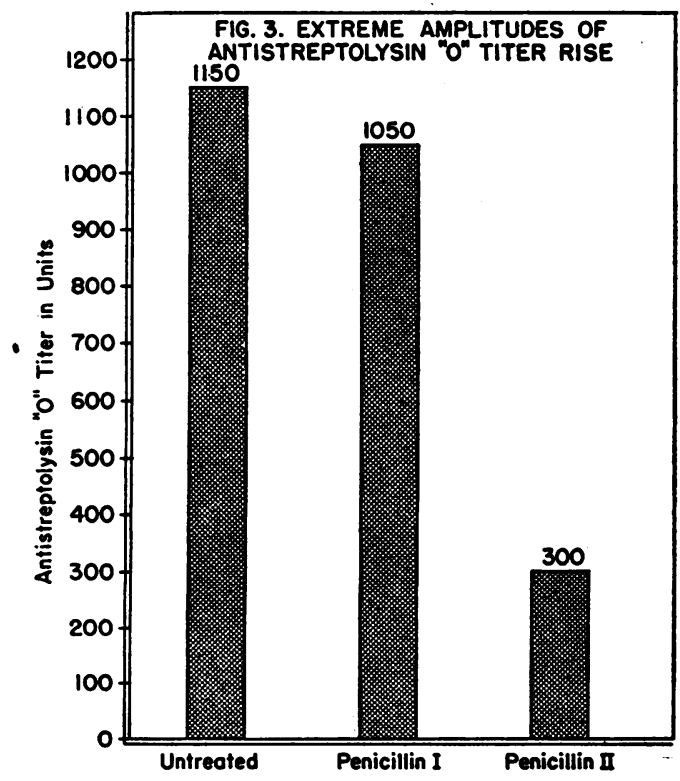

Penicillin I $=300,000$ units q.d. $\times 7,47$ patients Penicillin II $=20,000-50,000$ q. 3h. $\times 5.6$ days (ov.), 29 patients Untreated $=51$ patients

FIG. 3
Analysis of the untreated cases with high initial titers did not corroborate the finding of Rantz that the magnitude of rise was less in patients with high initial titers. The average rise in patients with initial levels of 200 or more units was 500 units, greater than the average rise in this group as a whole.

The apparent tendency of antimicrobic therapy to suppress the formation of antistreptolysin has been noted before. Rantz and his associates noted guardedly that an antistreptolysin response was observed less often in individuals receiving a long course of penicillin ( 80 hours), providing bacterial relapse did not occur, than with patients treated with short-course penicillin, sulfadiazine or salicylates (12). Earlier, Rantz, Kirby and Jacobs had presented "suggestive evidence that sulfonamide medication occasionally interfered with the development of agglutinins and antistreptolysin (13)." More recently, Weinstein and Tsao (7) have described suppression of the antistreptolysin formation in scarlet fever treated with a ten-day course of penicillin.

In analyzing the differing antibody responses with the two penicillin treatment schedules, it must be emphasized that although the 300,000 unit regimen (Penicillin I) provided a greater total dose and higher peak of serum concentrations, it effected detectable antibacterial levels for only a fraction of the 24-hour period. This is in contrast to the three-hourly dosage (Penicillin II), which maintained almost continuous detectable concentrations in the serum. It may be hypothesized that suppression of the antistreptolysin antibody by penicillin therapy is thus proportional to the duration of effective antibacterial action.

It is pertinent at this point to outline the rationale for the unusual penicillin dosage scheme denoted heretofore as Penicillin I.

Although the bulk of clinical and experimental evidence favors the use of multiple injections of penicillin in small dosage $(6,14), \mathrm{McD}$ ermott and his associates point out that "There is . . . reason to believe from clinical experience and from certain of the in vitro experiments that ... prolonged maintenance of an effective level need not be absolutely continuous (15)." Tillett et al., (16) have demonstrated that regimens utilizing widely spaced and small dosages of penicillin $(10,000$ units 
intramuscularly four times daily) will effect clinical improvement or cure in pneumococcal pneumonia. In fact, 100,000 units administered intravenously every three hours for three doses effected a "cure" for 30 hours although followed by a sharp return of symptomatology (16). Tillett's experience has received recent confirmation by Tompsett and McDermott who have successfully treated acute bacterial infections with large doses of penicillin in aqueous solution injected only once or twice daily (17). The present authors have successfully treated a case of proved pneumococcal pneumonia with single daily doses of 300,000 units given for seven days.

In this epidemic of an acute bacterial disease, conservation of the time of the overburdened nursing personnel was important, and a regimen entailing infrequent injections was therefore desirable. Secondary but important considerations were the elimination of the discomfort attendant upon multiple injections; and the opportunity to acquire further clinical experience with this type of therapy.

Moreover, in studying the effects of penicillin on antibody formation it seemed important to distinguish between the effects of continuous and sporadic dosage. 300,000 units was selected as an amount which would maintain detectable concentrations (greater than .078 units per cc.) in the blood stream for five to seven hours, an appreciable fraction of the 24-hour period(18).

\section{Significance of the antistreptolysin response}

It is generally accepted that a rise in the titer of antistreptolysin is indicative of a recent infection wtih the beta hemolytic streptococcus. The specificity of this response was established by the classical work of Todd $(1,19)$, which has been extensively confirmed. Mote and Jones (9) showed that 78 per cent of cases of scarlet fever had an antistreptolysin rise in convalescence. More recently, Rantz, Boisvert, and Spink (10) have described a significant antistreptolysin response in 87.5 per cent of 342 patients with streptococcal sore throat. All three groups of workers $(9,10$, 19) have also commented on the correlation of acute attacks of rheumatic fever and a rise in antisteptolysin titer. Similar rises have been noted in acute glomerulonephritis. Such evidence is basic to the present concept of rheumatic fever and glomerulonephritis as non-suppurative sequelae of streptococcal infection.

The significance of the suppression of the antistreptolysin antibody is conjectural. Weinstein noted that of seven untreated cases which showed no increase in antistreptolysin, two suffered recurrent streptococcal pharyngitis, and one, recurrent scarlet fever; and of 29 penicillin-treated cases with no rise in antistreptolysin titer, two had recurrences of streptococci in the pharynx and concomitant fever.

Five relapses occurred in the present series of 127 cases, all followed for a minimum of 21 days (Table I). Three were in the group receiving

TABLE I

Relapses*

\begin{tabular}{l|c|c}
\hline & Number of relapses & $\begin{array}{c}\text { Number with } \\
\text { antistreptolysin rise }\end{array}$ \\
\hline Untreated & 0 & 0 \\
Penicillin I (300,000 & 2 & $1 \dagger$ \\
units q.d.) & 3 & 0 \\
$\begin{array}{c}\text { Penicillin II (20,000- } \\
\text { 50,000 units q. 3 h.) }\end{array}$ & & \\
\hline
\end{tabular}

* The follow-up observation period in all cases was at least 21 days. "Relapse" means return of the clinical, laboratory, and bacteriologic picture of streptococcal pharyngitis.

$\dagger$ The second antistreptolysin titer was drawn following the relapse.

"regular" penicillin therapy (Penicillin II), and it is perhaps significant that none of these had had a rise in antistreptolysin titer prior to relapse. Moreover, two of these patients received no antibacterial therapy on re-admission to the hospital and on this occasion developed antistreptolysin rises of more than 300 and 1100 units, respectively. This fortuitous experiment demonstrated beyond doubt the ability of these patients to form antistreptolysin. Rantz, Boisvert and Spink have cautioned that evidence of antibody suppression must be interpreted in view of the "great individual differences which exist between various human beings in their ability to react to the antigenic stimulus of infection ... (12)."

The remaining two patients who showed clinical and bacteriological evidence of relapse had been treated with 300,000 units per day. Evaluation of these relapses in terms of antibody suppression is impossible as the final titers were not determined until after a second course of therapy. 
It has been mentioned that attacks of acute rheumatic fever, as well as other late sequelae of hemolytic streptococcal infection, are frequently associated with a rise in antistreptolysin titer. In Weinstein's study (7) all the cases of rheumatic fever and of glomerulonephritis occurred in patients who developed a rise in antistreptolysin titer.

Analysis of cases in our study who developed late complications reveals data in agreement with this past experience. Five patients developed definite evidences of late sequelae. Two had undoubted rheumatic fever, one, severe arthralgia with elevated sedimentation rate, one had microscopic hematuria, and another prolonged fever of 19 days' duration (Table II). All five patients de-

TABLE II

Late systemic sequelae

\begin{tabular}{l|c|c}
\hline \hline & Number and description of cases & $\begin{array}{c}\text { Number,with } \\
\text { antistrepto- } \\
\text { lysin rise }\end{array}$ \\
\hline Untreated & $\begin{array}{c}2 \\
\text { prolonged fever (1) } \\
\text { microscopic hematuria (1) } \\
3\end{array}$ & 2 \\
$\begin{array}{c}\text { Penicillin I } \\
(300,000 \text { units } \\
\text { q.d.) } \\
\begin{array}{c}\text { Penicillin II } \\
(20,000-50,000 \\
\text { units q. 3 h.) }\end{array}\end{array}$ & $\begin{array}{l}\text { rheumatic fever (2) } \\
\text { ? rheumatic fever (1) } \\
0\end{array}$ & 3 \\
\hline
\end{tabular}

veloped a rise in antistreptolysin titer. Three had been treated with intermittent high dosage penicillin while the two remaining cases were in the untreated group. No late sequelae have been observed to date in the group treated with sustained penicillin therapy.

\section{SUM MARY}

1. One-hundred-twenty-seven cases of hemolytic streptococcal pharyngitis were divided in rotation into three groups. One group served as an untreated control, one group was treated with large single daily doses of penicillin, and the third received virtually continuous penicillin therapy.

2. Antibody response, as measured by the antistreptolysin titers, was studied in all patients. The response varied in the three groups. In the untreated group the proportion of patients exhibiting a rise was comparable to that usually seen in streptococcal pharyngitis and scarlet fever. The antistreptolysin response in the group treated with continuous penicillin therapy was of appreciably lower frequency and magnitude than that manifested by the untreated group, as previously described by Weinstein and Tsao. Patients who recelved intermittent penicillin treatment manifested an incidence and degree of antibody response intermediate between that of the untreated and continuously treated groups.

3. All patients who developed late sequelae had manifested rises in antistreptolysin titer prior to or coincident with the appearance of these sequelae.

4. No patient who relapsed had developed a rise in antistreptolysin titer during the initial illness.

\section{CONCLUSIONS}

1. Penicillin therapy suppressed the formation of antistreptolysin in hemolytic streptococcal pharyngitis.

2. The degree of suppression of antistreptolysin formation was proportional to the duration of the antibacterial action of penicillin maintained during the 24-hour period. The antibody response was suppressed more by the maintenance of continuous effective penicillin blood concentrations than by the maintenance of effective penicillin levels for a fraction (five to seven hours) of the 24-hour period.

\section{BIBLIOGRAPHY}

1. Todd, E. W., Antigenic streptococcal hemolysin. J. of Exper. Med., 1932, 55, 267.

2. Commission on Acute Respiratory Diseases, Endemic exudative pharyngitis and tonsillitis. J. A. M. A., 1944, 125, 1163.

3. Rantz, L. A., Boisvert, P. J., and Spink, W. W., Hemolytic streptococcic and nonstreptococcic diseases of the respiratory tract; a comparative clinical study. Arch. of Int. Med., 1946, 78, 369.

4. Loge, J. P., and Kilbourne, E. D., Penicillin treatment of streptococcal pharyngitis. To be published.

5. Plummer, N., Duerschner, D. R., Warren, H. D., Rogliano, F. T., and Sloan, R. A., Penicillin therapy in hemolytic streptococcic pharyngitis and tonsillitis. J. A. M. A., 1945, 127, 369.

6. Rammelkamp, C. H., and Kirby, W. M., Factors determining the dosage of penicillin in the treatment of infections. Bull. New York Acad. Med., 1945, 21, 656.

7. Weinstein, L., and Tsao, C. L., Effect of types of treatment on development of antistreptolysin in patients with scarlet fever. Proc. Soc. Exper. Biol. \& Med., 1946, 63, 449. 
8. Rantz, L. A., Boisvert, P. J., and Spink, W. W., Hemolytic streptococcus sore throat: A detailed study of the simultaneous infection of a large number of men of a single type. Arch. Int. Med., 1945, 76, 278.

9. Mote, J. R., and Jones, T. D., Studies of hemolytic streptococcal antibodies in control groups, rheumatoid arthritis. II. The frequency of antistreptolysin "O," antifibrinolysin and precipitating-antibody responses in scarlet fever, hemolytic streptococcal infections and rheumatic fever. J. of Immunol., 1941, 41, 61.

10. Rantz, L. A., Boisvert, P. J., and Spink, W. W., Etiology and pathogenesis of rheumatic fever. Arch. Int. Med., 1945, 76, 131.

11. Rantz, L. A., Group A hemolytic streptococcus antibodies. III. A study of the simultaneous infection of a large number of men by a single type. Arch. Int. Med., 1944, 73, 238.

12. Rantz, L. A., Boisvert, P. J., and Spink, W. W., Hemolytic streptococcal sore throat; antibody re- sponse following treatment with penicillin, sulfadiazine and salicylates. Science, 1946, 103, 352.

13. Rantz, L. A., Kirby, W. M., and Jacobs, A. H., Group A hemolytic streptococcus antibodies. I. Griffith Type agglutinin and antistreptolysin titers in normal men and in acute infections. J. Clin. Invest., 1943, 22, 411.

14. Anderson, Donald G., Medical progress; the treatment of infections with penicillin. New England J. Med., 1945, 232, 400.

15. McDermott, W., Benoit, M., and DuBois, R., Timedose relationships of penicillin therapy. III. Regimens used in early syphilis. American J. of Syph., Gonor. \& Ven. Dis., 1945, 29, 345.

16. Tillett, W. S., McCormack, J. E., and Cambier, M. J., Treatment of lobar pneumonia with penicillin. .J. Clin. Invest., 1945, 24, 589.

17. McDermott, W., Personal communication.

18. Tompsett, R., Personal communication.

19. Todd, E. W., Antihemolysin titres in hemolytic streptococcal infections and their significance in rheumatic fever. Brit. J. Exper. Path., 1932, 13, 248. 